\title{
Dimensional Structures of Human Societies: An Alternative Interpretation of Agoramétrie-Type Survey Results
}

\author{
Warren D. TenHouten \\ Department of Sociology, University of California, Los Angeles, USA \\ Email:wtenhout@g.ucla.edu
}

How to cite this paper: TenHouten, W. D. (2018). Dimensional Structures of Human Societies: An Alternative Interpretation of Agoramétrie-Type Survey Results. Sociology Mind, 8, 154-167. https://doi.org/10.4236/sm.2018.82013

Received: February 18, 2018

Accepted: April 25, 2018

Published: April 28, 2018

Copyright $\odot 2018$ by author and Scientific Research Publishing Inc. This work is licensed under the Creative Commons Attribution International License (CC BY 4.0).

http://creativecommons.org/licenses/by/4.0/

\begin{abstract}
Surveys of social and political issues using Agoramétrie methodology drawn from widely different social environments have revealed consistent two-dimensional, factor-analytic solutions. Rukavishnikov and van Meter refer to the first dimension as "modern-radical/traditional-conservative" and "openness/closedness"; the second, as "frustration/satisfaction" and "emotional/non-emotional". These distinctions are critically evaluated and an alternative interpretation is proposed. Two sociorelational dimensions are drawn from Douglas's Grid-Group Theory, interpreted as two pairs of opposite social-relations models of Fiske-Haslam-Bolender Relational Models Theory. Accordingly, the Group emphasizes communal-sharing (CS) as opposed to market-pricing (MP); the Grid, authority-ranking (AR) as opposed to equality-matching (EM). Affect-Spectrum Theory links valenced, secondary-level emotions to quadrants of the Grid-Group space, with quadrants characterized by sets of secondary-level emotions. Van Meter's hypothesis that the two-dimensional survey results suggest two kinds of human societies, the "cooperative" and the "hierarchical" is reasonable, but it is proposed that the sociorelational bases of these societal types are the complementarities between CS and EM, and between AR and MP. Neurosociological implications of the data and theorizing are discussed.
\end{abstract}

\section{Keywords}

Opinion Polling, Grid-Group Theory, Relational-Models Theory, Affect-Spectrum Theory, Emotion

\section{Introduction}

Are there basic dimensions of human societal-level social organization? A pro- 
gram of research conducted by the Parisian association of administrations and companies Agoramétrie $(1987,1998)$ suggests a possible two-dimensional structure underlying the sociocognitive organization of social groups or societies. The naming of these two "factors" differs considerably, and their justification in terms of the placement of questionnaire-item responses in this factor-analytic space is based on thin evidence. We present an alternative interpretation of these two axes, based on a partial synthesis of three social theories, Grid-Group Theory (Douglas, [1970] 2003: pp: 64-106; Gross \& Rayner, 1985), Relational-Models Theory (RMT) (Fiske, 1991, 2004; Haslam, 1994a, 1994b; Bolender, 2010), and Affect-Spectrum Theory (TenHouten, 2007, 2013, 2017b).

This paper, in offering a theoretical interpretation of these two-factor solutions, proposes that both axes contain not one but two dimensions. We hypothesize that both axes are formed from a polarity of opposite sociorelational models. One axis is interpreted as Douglas' "group" and as the difference between the sociorelational models Communal Sharing (CS) and Market Pricing (MP); the other axis, as Douglas's "grid" and the difference between social hierarchy (Authority Ranking) (AR) and social equality, equity, and fairness (Equality Matching) (EM). We then extend the analysis to the four quadrants derived from the poles of these paired dimensions and use an emotions classification system to identify four key emotions linked to each quadrant. We also briefly discuss the place of these concepts with respect to evolutionary neurosociology. Before presenting this theoretical conceptualization, we discuss and evaluate existing interpretations.

\section{The Two Principal Components Axes of Agoramétrie-Type Datasets}

For three decades, Agoramétrie researchers have conducted annual surveys (excepting 1979 and 1980) of public opinion, sampling both the French population and the media discourse about contemporary social issues and social conflicts. From these two sources, closed-ended questionnaires based on sampling of ongoing issues are constructed, and data are obtained through face-to-face interviewing are then analyzed, typically using principal-components factor analysis. There are sociologically-important findings derived from comparative analyses of results from studies following this Agoramétrie methodology. First, across different populations, nations, cultures, economic situations, war or peace, and changing environmental conditions, a common core of about 30 - 40 "trunk" questions occur (used together with about 50 - 70 other questions), suggesting that some issues are likely cross-culturally universal, being fundamental, perennial issues of concern in human societies. And second, the first and second principal-component axes of these investigations are quite stable across studies, and might reflect fundamental "structures of human societies," possibly meaning that there are two basic types of human society (van Meter, 2014).

Two-dimensional solutions repeated occur in analyses of Agoramétrie-type surveys of contemporary sociopolitical problems and issues. There has been 
considerable overlap in naming these two factors across independent investigations. We focus on one of these two-dimensional plot, shown as Figure 1, from a Russian opinion study conducted in 1991-1992 by Rukavishnikov (1992), who interprets the first axis of his factor analysis as an opposition between "modern-radical" vs. "traditional-conservative," the second as "satisfaction" vs. "frustration". These dimensions are contrasted as "ideological" and "material," corresponding to Marx's (1859) duality of "superstructure" and "base".

Reviewing this and other Agoramétrie-method studies of social issues and social conflicts, van Meter (2001, 2004, 2014: p. 39) employs a different terminology, referring to these two axial dimensions as "openness" vs. "closedness," and "non-emotional" vs. "emotional," respectively. Here, we focus on Rukavishnikov's (1992) factor-analytic plot, shown in Figure 1. Comparison of the left and right sides of this plot, factor 1, lends only limited support to the distinction between left-wing or radical, and right-wing, or conservative, orientations; on the right side of the plot, we find phrases such as "God exists", "traditional", and "conservative". On the radical-leftist side, we find terms such as "modernist", "trust in new politics", and "army outside the politic".

According to Rukavishnikov, the second, vertical factor contrasts "satisfaction" (below) and "frustration" (above), which, according to Basic Psychological Need Theory (Ryan \& Deci, 2000; Deci \& Ryan, 2000, 2012; Chen et al., 2015), are affective responses to psychological needs for autonomy, relatedness, and competency. Items in the lower half of this plot expresses "feeling secure", "OK", "pro", and "adventure", which vaguely suggest satisfaction. Items at the top possibly reflect frustration in the terms, "challenge", "contra", and "anti-", "split", and "cancel". However, there is overlap, as the top-half terms "trust", "pro", and "confident" are positively affect-laden and hardly expressions of frustration, and, in the bottom half, there would appear to be little satisfaction in "submission".

Parallel to Rukavishnikov's "radical"-“conservative distinction," van Meter (2014) uses the alternative terms, "openness"- "closedness," respectively. While in general the "radical" items of Figure 1 are to the left and the "conservative" items to the right, there is some overlap: Two terms that possibly suggest a leftist orientation occur on the right side ("anti-Western", "reestablish USSR"), and three terms suggestive of a rightist ideology are on the left side ("orthodox state religion", "pro private property", "pro death penalty"). There is obviously more to the vertical axis of Figure 1 than needs being met, and more to the horizontal axis than political ideology and open- or closed-mindedness (see Rokeach, 1960).

Our thesis is that there exists a more general way to interpret these same two axes. We hypothesize that these axes refer not just to psychological needs and political beliefs, but to underlying social-relations models. Social and behavioral scientists widely agree that social relations are facilitated by mental structures, or schemas, which individuals use to organize and interpret social information and coordinate interactions with others as they navigate the social world. One such 


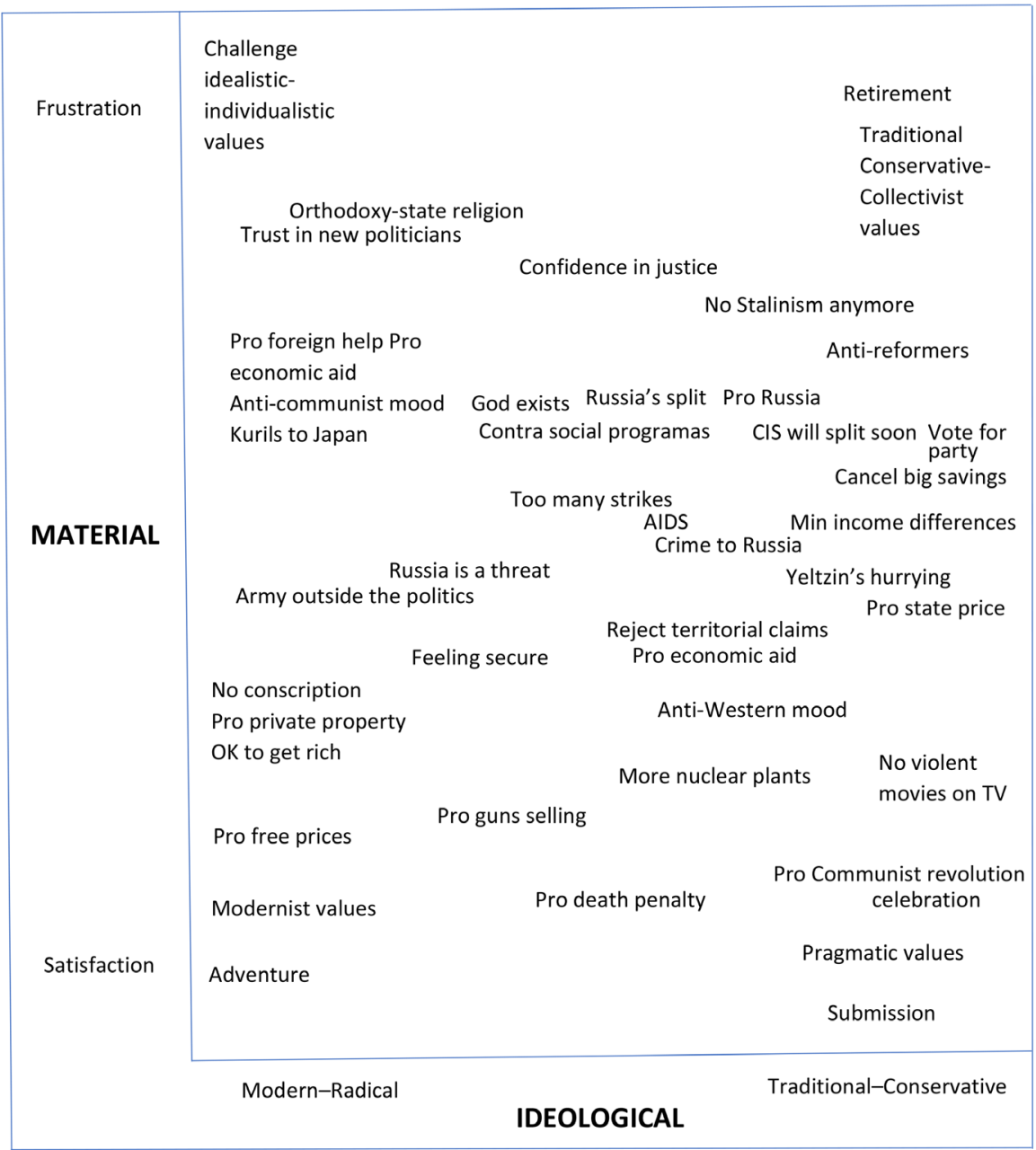

Figure 1. Russian public opinion structure (Rukavishnikov, 1992) - a plot of the first principal components.

theory-the cognitive-anthropological relational-models theory (Fiske, 1991), posits a cross-cultural set of four elementary relational models-CS, EM, AR, and MP. These relational models exist pan-culturally, possibly because they have been evolutionarily adaptive.

Communally-shared social relations centrally involve birth, begetting, and the institutions of family and kinship, and are validated through connection to the past, through tradition, ceremony, rite, ritual, and law. They are "based on duties and sentiments generating kindness and generosity among people perceived to be of the same kind, especially kin" (Fiske, 1991: p. 14). CS involves a group entitativity, a "we-mode" (Gallotti \& Frith, 2013) based on solidarity, social cohesion, and "a subjective feeling of the parties, whether affectual or traditional, that they belong together" (Weber, [1921] 1978: p. 40).

Equality-matched social relations involve turn-taking, quid pro quo behavior, distributive justice, and in-kind reciprocity. "Giving back" is proportional to what one receives in all situations (Laursen \& Hartup, 2002), including those involving conflict, where EM matching can take the form of eye-for-an-eye reta- 
liatory vengeance in accordance with lextalionis. In decision making, EM obtains when everyone has one vote or an equal voice. In equality-matching, individuals "conceive of each other-or the rights, duties or actions involved in the relationship as distinct, but as balancing each other, aligning, or matching, so they are interchangeable," and "[p]ersons are intersubstitutable in...that they match and correspond on an even basis" (Fiske, 1991: p. 15; see also Fiske, 2004: pp. 103-111).

Authority-ranked social relations involve a cluster of closely related concepts, including social power, domination, authority, influence, status, and prestige (Haugaard, 2010). Social dominance hierarchies exist because there are social, personal, biological, and physical rewards for prevailing in competitive situations. On the societal level, hierarchies emerge because pressing problems require institutions of decision making regarding protection and aggressive resource acquisition.

Market-pricing social relations involve the production, acquisition and consumption of resources and control of territory, and involve the behaviors of production, distribution, consumption, and resource maintenance. Economic exchange relies on quantification of the values of resources, objects, and social positions. In exchange-based relationships, individuals "denominate value in a single metric, typically price" (Fiske, 1991: p. 15).

\section{An Alternative Interpretation of the Two Principal-Component Axes}

The horizontal axis of Figure 1 shows a contrast between individualism and collectivism, which corresponds, at least roughly, to MP and CS. On the left side of the plot, we find terms such as "individualistic", "economic", "private property", "prices", and "anti-communist". On the right side of this figure, we find an opposite, CS-related set of terms, including "traditional", "collectivist", "for party", "celebration", and "pro-communist". Thus, the second polarity would appear to be interpretable as "MP-CS", which suggests this axis contains not one but two dimensions.

The vertical axis, satisfaction-frustration (or for van Meter, "non-emotional"- "emotional") we conceptualize as a polarity between social inequality, or social power, and an egalitarian orientation. This corresponds to the contrast between the social-relational models $\mathrm{AR}$ and EM. In the top two-thirds of Figure 1, we find words expressing social relations of authority or forcefulness, including "state", "claims", "anti-", "justice", "cancel", "re-establish", and "violent". In the bottom third of this figure, we find a more meagre list, of "free prices", "pro death penalty" (an example of quid pro quo justice, indicative of EM), and "pro-communist" (if seen as a Marxian utopian ideal). EM and AR are opposites, as one prizes equality while the other is based on inequality, which are opposed even logically (If $(\mathrm{A}=\mathrm{B})$, then $\mathrm{A}>\mathrm{B}$ or $\mathrm{A}<$ B.). We call the vertical axis "AR-EM", which is a measure of social power, but is a function of two dimensions. We next elaborate the social-relations variables used to reinterpret the two axes of opinion. 


\section{Quadrant Analysis: The Two Axes, Grid-Group Theory, and Relational-Models Theory}

Studies of Agoramétrie-type plots have found that after dichotomizing both axes, the resulting quadrants lend themselves to interpretation. For example, in his plot of "trunk" questions, van Meter (2014: p. 78) describes a kind of individual who feels "insecure", would "bring back the death penalty", and feels there are "too many immigrant workers", suggesting that, "The reader can easily imagine what sort of person would hold this network of opinions and what other positive and negative ties with other opinions would likely exist" (van Meter, 2014: p. 38).

Given the present interpretation of the two discovered axes as functions of the oppositions between CS and MP, and between AR and EM, we might inquire as to continuities between the two-dimensional plots and efforts to measure culture by classifying relational-model orientations in the four quadrants. To this end, we first propose unification of Douglas's Grid-Group Theory and Relational-Models Theory (Fiske, 1991). For Douglas, the "group" represented the extent to which community members are highly involved in informal social relations, an extreme case being "a monastic or communal setting," and more generally, a social situation where there is "long term commitment and a tight identification of members with one another as a corporate entity", and where "individual are expected to act on behalf the collective whole" (Gross \& Rayner, 1985).

At the other end of this horizontal axis continuum, we find individualism, where societal members act in their raw self-interest, not being constrained by, or reliant upon, the group or community. "The low group experience is a competitive, entrepreneurial way of life where the individual is not strongly constrained by duty to other persons" (Gross \& Rayner, 1985: p. 6). Thus, the horizontal axis of Figure 1, which have been interpreted as open-closed and satisfied-frustrated, are here given a new interpretation, as "group = communal-sharing satisfied-frustrated market-pricing".

The vertical axis, as Douglas's grid, can be interpreted as a dimension of social power, with AR at the high and EM at the low ends of the second factor. The grid is described by as "a complementary bundle of restraints on social interaction", where "roles are distributed based on...social classifications such as...position in a hierarchy, holding a bureaucratic office, descent in a senior clan or lineage, or point of progress through an age-grade system". A low-grid social environment is one in which "access to roles depends upon personal characteristics..., or even of formal regulations for taking equal turns" (Gross \& Rayner, 1985: p. 6).

The low-group-low-grid, MP-EM condition corresponds to the lower-left quadrant of Figure 1; it describes a person characterized in neoclassical economics as a perfect competitor, who can be described by "pro free prices," "pro private property", "OK to get rich", "pro guns selling", and endorsing "adventure" (or resource-seeking enterprise).

The low-group-high-grid person endorses "individualistic values", being 
"contra social programs", and disapproves of collective action ("too many strikes"), which are consistent with the low-grid, EM-oriented social orientation. At the same time, such a person is oriented to social hierarchy, to the high-grid-AR condition, as expressed in "orthodoxy-state religion", "God exists", "anti-communism", and in being "contra social programs".

In the upper right, high-grid-high-group, AR-CS quadrant, we again find endorsement of social hierarchy, as reflected in the terms "traditional", "conservative", and "anti-reformers", and political loyalty ("vote for party"). The CS-based terms in this quadrant are few, but clear in the meaning of "collectivist values" and, possibly, in being "pro Russia” yet wanting "no Stalinism.”

\section{Emotions and Social Relations}

According to van Meter (2014), the second axis of social organizational is distinguished as emotional and non-emotional. There is no doubt that some individuals experience negative emotions, even to the point of despair and the collapse of the right hemisphere, and with it, of the self (Weinberg, 2000; TenHouten, 2017a), whereas individuals with a left-hemisphere orientation have more positive affect. This distinction, as van Meter (2014) observes, has been established by Davidson (1998) and other neuroscientists. Yet, it is not the radical-conservative, openness-closedness dimension that Agoramétrie-Type classification see as affective, but rather the second, satisfaction-frustration, non-emotional-emotional dimension that is affective. The alternative interpretation of the two axes is helpful at this point. Affect-spectrum theory (TenHouten, 2007, 2013, 2017b) holds that the existential problems of temporality, identity, hierarchy, and territoriality have given rise to CS, EM, AR, and MP, respectively. Since all emotions are adaptive reactions to sociorelational circumstances as shown in Plutchik's ([1962] 1991) model of the primary emotions (Figure 2), we expect that negative and positive experiences of these four social relations will have prototypical, basic emotional reactions, and indeed $\mathrm{CS}+\rightarrow$ joy/happiness, CS- $\rightarrow$ sadness, EM $+\rightarrow$ acceptance, EM- $\rightarrow$ rejection/disgust, $\mathrm{AR}+\rightarrow$ anger, $\mathrm{AR}-\rightarrow$ fear, $\mathrm{MP}+\rightarrow$ anticipation/exploration, and $\mathrm{MP}-\rightarrow$ surprise. We expect to find secondary-level emotions in the four quadrants, that emotions can be found both at the top and the bottom of the second factor, and that all four quadrants contain both positively-, mixed-, and negatively-valenced emotions concentrated in all four quadrants, as shown in Figure 3.

Prediction of these emotions would provide a test of the sociorelational interpretation of the Agoramétrie-based model of the structure of human societies. One such test, locating pride and shame in the upper right (CS-AR) quadrant, provides a model for such theory testing (TenHouten, 2017c). As hypothesized, in this study is was found that pride, defined as an angry joy, was predicted by $\mathrm{AR}+$ and $\mathrm{CS}+$, whereas shame, a fearful sadness, was predicted by AR- and CS-.

Of these 16 emotions, only four (aggressiveness, pride, fatalism-more generally, resourcefulness, and love) are approach-oriented in that their component 


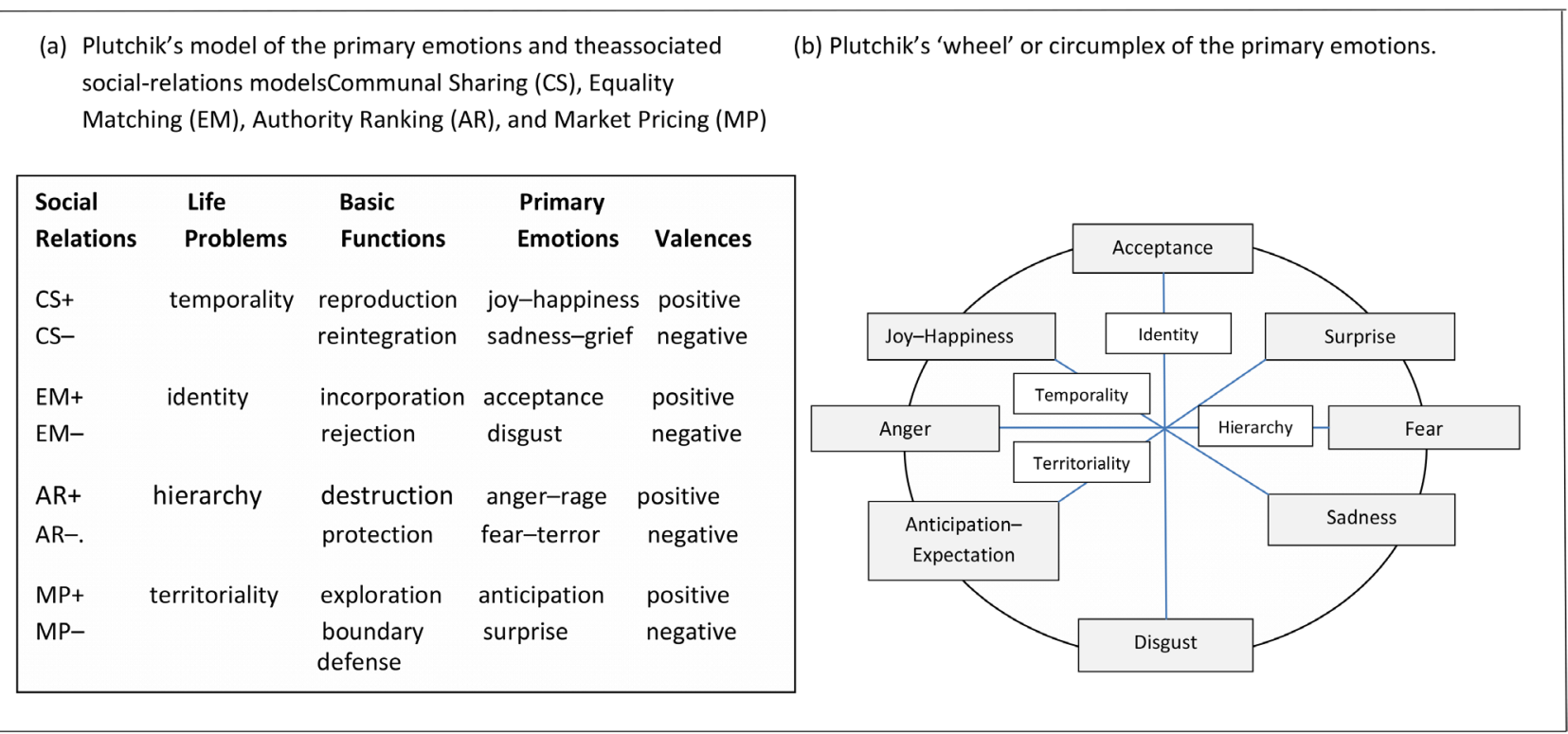

Figure 2. (a) Social-relations models, life-problems, basic functions, primary emotions; (b) Plutchik's circumplex or "wheel”.

\begin{tabular}{|c|c|c|}
\hline \multirow{4}{*}{$\begin{array}{l}\text { Authority } \\
\text { Ranking }\end{array}$} & Aggressiveness = Anticipation & Pride $=$ Anger \& Joy \\
\hline & \& Anger $\leftarrow \mathrm{MP}+\& \mathrm{AR}+$ & $\leftarrow \mathrm{AR}+\& \mathrm{CS}+$ \\
\hline & $\begin{array}{l}\text { Anxiety = Anticipation \& Fear } \\
\leftarrow \mathrm{MP}+\& \mathrm{AR}-\end{array}$ & Sullenness = Anger \& \\
\hline & $\begin{array}{l}\text { Outrage = Surprise \& Anger } \\
\leftarrow \mathrm{MP}-\& \text { AR+ }\end{array}$ & $\begin{array}{l}\text { Guilt = Fear \& Joy } \\
\leftarrow \text { AR- \& CS }+\end{array}$ \\
\hline \multirow{2}{*}{ GRID } & $\begin{array}{l}\text { Alarm = Surprise \& Fear } \\
\leftarrow \mathrm{MP}-\& \mathrm{AR}-\end{array}$ & $\begin{array}{l}\text { Shame }=\text { Fear \& Sadness } \\
\leftarrow \mathrm{AR}-\& \mathrm{CS}-\end{array}$ \\
\hline & $\begin{array}{l}\text { Fatalism = Anticipation \& } \\
\text { Acceptance } \leftarrow \mathrm{MP}+\& \text { EM+ }\end{array}$ & $\begin{array}{l}\text { Love = Acceptance \& Joy } \\
\leftarrow \mathrm{EM}+\& \mathrm{CS}+\end{array}$ \\
\hline \multirow{7}{*}{$\begin{array}{l}\text { Equality } \\
\text { Matching }\end{array}$} & $\begin{array}{l}\text { Cynicism = Anticipation \& } \\
\text { Disgust } \leftarrow \text { MP + \& EM- }\end{array}$ & $\begin{array}{l}\text { Resignation = Acceptance \& } \\
\text { Sadness } \leftarrow \text { EM }+\& \text { CS- }\end{array}$ \\
\hline & Curiosity = Surprise \& & Derisiveness = Disgust \& Joy \\
\hline & Acceptance $\leftarrow \mathrm{MP}-\& \mathrm{EM}+$ & $\leftarrow \mathrm{EM}-\& \mathrm{CS}+$ \\
\hline & Shock = Surprise \& Disgust & Loneliness = Disgust \& \\
\hline & $\leftarrow \mathrm{MP}-\& \mathrm{EM}+$ & Sadness $\leftarrow$ EM $-\&$ CS- \\
\hline & \multirow[t]{2}{*}{ Market Pricing } & Communal Sharing \\
\hline & & GROUP \\
\hline
\end{tabular}

Figure 3. The sociorelational dimensions of Grid and Group, and the four secondary level emotions of the quadrants.

primary emotions and associated social relations models are positively valenced. Their opposites-alarm, shame, shock, and loneliness, are withdrawal-oriented, 
having only negative components and relational models. The other eight are of mixed valence, and from a psychological point of view are typically experienced as negative. With respect to the satisfaction-frustration distinction, we cannot say that the emotions in the top half of this conceptual space are frustrating, and that the rest are satisfying. And as for the emotional-non-emotional distinction, all four quadrants of the space involve emotions. Thus, the interpretation of the two axes offered here leads to a very different view of the second dimension.

\section{Two Kinds of Society?}

Van Meter (2014) speculates that if there are two dimensions structuring human society, then there might well be two kinds of society. He refers to these putative societal as "cooperative" and "hierarchical". In periods of calm, with abundant resources, the most adaptive societies emphasize cooperation and openness, as opposed to hierarchy and closedness. For societies threatened, with their survival at stake, hierarchy can emerge. Thus, there are at least two main means of managing social conflict (van Meter, 2001).

Our four social relations models have been grouped as opposites (CS-MP as group, AR-EM as grid). Here, we rearrange these four social relations models as a pair of complementarities, of $\mathrm{CS} \cap \mathrm{EM}$ and $\mathrm{AR} \cap \mathrm{MP}$, and see these as the bases of what van Meter (2014) calls "cooperative" and "hierarchical", corresponding to the ordinary distinction between "community" and "society" (Tönnies, 1887), also to the difference between "hedonic" and "agonic" kinds of social organization (Chance, 1988). Our hypothesis is that cooperative-community-hedonic social organization is based on the two relational models CS and EM, and AR and MP, respectively.

Equality-matching and communal-sharing form the basis of a second type, 'hedonic community'. Individual gain a sense of community in perceiving they are all the same, have common knowledge of the state of their community, and have a far-ranging like mindedness, sharing the same beliefs and core values. On the other hand, CS involves more than goods and things, for it is important that information about the state of the community, the "word" about ongoing social events and processes, that when commonly perceived is in and of itself a form of EM. Also, the shared perception that communal resources can be used by individuals as need arises, leads quite naturally to the idea that favors and gifts should roughly be reciprocated, and access to communal resources, open to all, is in and of itself a form of social equality.

Within communities, interpersonal relations are heterocentric, and members center interest, attention, and value on others, thereby attaining a self-realization of the personal in which they are related as equals. This does not mean they have equal abilities, rights, social statuses, or any other de facto equality; their equality is rather an aspect of the mutuality of the relationship, in which participants do not constrain one another and act consensually. Communal relations are go- 
verned by a norm of reciprocal generosity and gratitude (Simão \& Seibt, 2014), which requires a social morality holding that debt and favors be repaid to retain balance and equality. "To maintain equality of persons in relation is justice", requiring that individuals' interests be perceived of as being considered equally with those of all other community members (Macmurray, 1999: p. 190). Thus, communal relations can be equalized in requiring for all freedom of action, and respecting the autonomy of others, with the constraint that other not be injured or deprived of what they might need; this requires agreement about what is perceived of as fair and just with respect to what belongs to the community. Such communal sharing can be based on a principle of egalitarian distribution of resources, so that all community members are perceived of as entitled to an equal share of resources, or at least to an equality of opportunity for acquiring resources.

Chance (1988) described "agonic" social organization as competitive, conflictual, and hierarchically organized around the principle of social dominance and associated control of resources. We refer to this AR-MP combination as the secondary relational model "agonic society". Social dominance is conceptualized both in terms of rank in the social hierarchy and the capacity to acquire and control resources. Any two individuals in agonic society are either of the same or different social rank, and all are concerned with self-security and maintaining, or improving, their status levels. In primate societies, life possesses a continual uncertainty, because social ranking is actively and aggressively contested. Lower-ranking members live with anxiety, execute gestures of appeasement and submission, and are fearfully poised to escape the wrath and punishment of higher ranking members. Social control is expressed territorially, through the physical closeness of the lower-ranking members to the centrally dominant figure(s). Thus, in agonic society, hierarchy is inseparable from, and is articulated in terms of, territory. Hierarchy and territoriality are the bases of AR- and MP-based social relations (TenHouten, 2013: pp. 31-35). As small-scale egalitarian societies increase in size and complexity, social dominance structures emerge and become institutionalized, so that communal resources become subject to allocation based not on any principle of equality but on status, prestige, and power. This requires social cognition, and social intelligence, both for this subject to power and those who wield power (Hallpike, 1988: pp. 208-287).

\section{Discussion}

Van Meter (2014: p. 40) links openness-closedness to an emotional distinction between approach and withdrawal, with positive affect related to positive affect and activation of left hemisphere (LH) frontal lobe activity, and with withdrawal linked to right hemisphere (RH) comparatively negative affect (Davidson, 1998). Each hemisphere has a different affective style, but the link between satisfaction-approach for the $\mathrm{LH}$, and frustration-withdrawal for the $\mathrm{RH}$ are difficult to reconcile with the open-closed distinction, because it is the RH, which we 
might associate with social cooperativeness, that is open and curious with respect to all kinds of information, whereas the LH prefers to focus on its own dynamics and is therefore relatively closed, yet is oriented to hierarchical classification (McGilchrist, 2010). Sylvan Tomkins' (1957) left- and right-wing ideological polarity scale has been studied by Ashton \& Dwyer (1975); see also Stone, (1986), who found that left-wing ideologues' conjugate-lateral eye movements were predominantly leftward (indicating right-hemisphere activation), and as gestalt-synthetic mode of processing that is open to all kinds of information); right-wing ideologues, in contrast, were predominantly right-lookers (indicating left-hemisphere activation, and a logical-analytic mode of information processing resistant to interruptions). Thus, there is some evidence of consistency in the structural equation: "radical: openness: right-hemisphericity: conservative: closedness: left-hemisphericity".

The findings for the second dimension of the Agoramétrie-type survey factor analyses would appear to be on weak grounds. There is virtually no support for Rukavishnikov's (1992) interpretation of this dimension as satisfaction-frustration, and this cognitive-affective distinction is difficult to see as involving the economic 'base' of society. Nor is there support for van Meter's parallel distinction between the non-emotional and the emotional. Our alternative, $\mathrm{AR}-\mathrm{EM}$ and $\mathrm{CS}-\mathrm{MP}$ dimensions inferred to correspond to the grid-group distinction, together with propositions from affect-spectrum theory, suggest that both positive, mixed, and negative emotions can be found within all four quadrants.

While Agoramétrie-type survey analysis has led to interesting generalizations, and fueled theoretical speculation, clearly the questions raised cannot be answered by the same method that enabled them to be framed. Insofar as there is interdependence between elementary social relations models-which are essentially mental representations of the social, the mental realms of cognition and emotion, and brain organization, then studies of the sociohistorical development of societies, and the development of social relations and associated mental structures in individual, require neurosociological research and theory.

\section{Conclusions}

The Agoramétrie methodology has produced rich data that has been analyzed using an inductive method, primarily factor analysis. Studies using this method, in different societies and conditions, have consistently produced two-factor solution, indeed a fascinating result, which has led to the hypothesis of a twofold dimensionality of human society. While there is continuity in results, there is wide discrepancy in the naming of the factors, as illustrated by the very different interpretations of the two axes by van Meter and Rukavishnikov. The problem is that there is no way, with available data considered inductively, to determine if the two axes are single dimensions or two-dimensional polarities.

The only solution is through sociological theorizing the dimensional structure of the social order, and then, deductively, interpreting the two axes. While theo- 
retical models in philosophy and social science vary in their dimensionality, four dimensional solutions have been ubiquitous, and are typically formulated as intersections of two basic polarities or dualisms (Harmon, 2009). By dichotomization the four relational models and defining the four quadrants as $A R \cap C S, A R$ $\cap \mathrm{MP}, \mathrm{EM} \cap \mathrm{CS}$, and $\mathrm{EM} \cap \mathrm{MP}$, we define four secondary-level relational models. From the emotions classification provided by affect-spectrum theory, we infer the emotions most directly involved in social relations. There is an obvious advantage to considering specific emotions as opposed to distinction such as frustration-satisfaction and emotional-non-emotional. Satisfaction and frustrations are indeed important, but they are not emotions, and we find emotions that offer satisfaction in every quadrant, for there is satisfaction in self-assertion, or aggression, pride, fatalism (more generally, resourcefulness), and love. As for the emotional-non-emotional distinction, we suggest that every kind of (valenced) social relation calls upon a set of specific emotions, so there is no area of a sociorelational space that is devoid of affect.

That secondary emotions of positive, mixed, or negative valence can be linked to the four quadrants follows from the fact that elementary social relations are themselves valenced. Relational-models theory can be criticized for ignoring situations of conflict and contention. For example, Haslam (1994a) develops scales only for the positively-valenced relational models.

We need to consider not only happy families, social equality, legitimate authority, and wealth but disrupted place in community, inequality, subordination and exploitation, and poverty. Thus, while there are indeed four elementary social relations that can model society, and possibly the evolution of society, there are not four but eight elementary forms of sociality and these and the emotions to which they are causally linked and would appear to exist as natural kinds.

\section{References}

Agoramétrie (1987). Les structures de l'opinionen 1985-Enquêtesetméthodologie. Bulletin of Sociological Methods/Bulletin de Méthodologie Sociologique, 14, 94-97.

Agoramétrie (1998). Les Structure de l'opinion fin 1997. Paris: Agoramétrie.

Ashton, V. L., \& Dwyer J. H. (1975). The Left: Lateral Eye Movements Andideology. Perceptual and Motor Skillsm, 41, 248-250. https://doi.org/10.2466/pms.1975.41.1.248

Bolender, J. (2010). The Self-Organizing Social Mind. Cambridge, MA: The MIT Press. https://doi.org/10.7551/mitpress/9780262014441.001.0001

Chance, M. R. A. (1988). "Introduction" and “A System Synthesis of Mentality”. In M. R. A. Chance (Ed.), Social Fabrics of the Mind (pp. 1-45), Hove: Lawrence Erlbaum.

Chen, B., Deci, E. L., Matos, L., \& Soenens, B. (2015). Basic Psychological Need Satisfaction, Need Frustration, and Need Strength across Four Cultures. Motivation and Emotion, 29, 216-236. https://doi.org/10.1007/s11031-014-9450-1

Davidson, R. J. (1998). Affective Style and Affective Disorders: Perspective from Affective Neuroscience. Cognition and Emotion, 12, 307-330. https://doi.org/10.1080/026999398379628

Deci, E. L., \& Ryan, R. M. (2000). The "What" and "Why" of Goal Pursuits: Human Needs and the Self-Determination of Behavior. Psychological Inquiry, 11, 227-268. 
https://doi.org/10.1207/S15327965PLI1104_01

Deci, E. L., \& Ryan, R. M. (2012). Motivation, Personality, and Development within Embedded Social Contexts: An Overview of Self-Determination Theory. In R. M. Ryan (Ed.), Oxford Handbook of Human Motivation (pp. 85-107), Oxford: Oxford University Press.

Douglas, M. [1970] (2003). Natural Symbols: Explorations in Cosmology. London: Routledge.

Fiske, A. P. (1991). Structures of Social Life: The Four Elementary Forms of Humanrelations. New York: Free Press.

Fiske, A. P. (2004). Relational Models Theory 2.0. In N. Haslam (Ed.), Relational Models Theory: A Contemporary Overview (pp. 3-25), Mahwah, NJ: Lawrence Erlbaum.

Gallotti, M., \& Frith, C, D. (2013). Social Cognition in the We-Mode. Trends in Cognitive Science, 17, 160-165. https://doi.org/10.1016/j.tics.2013.02.002

Gross, J. L., \& Rayner, S. (1985). Measuring Culture: A Paradigm for the Analysis of Social Organization. New York: Columbia University Press.

Hallpike, C. R. (1988). The Principles of Social Evolution. Oxford: Clarendon Press.

Harmon, G. (2009). Dwelling with the Fourfold. Space and Culture, 12, 292-302. https://doi.org/10.1177/1206331209337080

Haslam, N. (1994a). Categories of Social Relationship. Cognition, 53, 59-90. https://doi.org/10.1016/0010-0277(94)90077-9

Haslam, N. (1994b). Mental Representation of Social Relationships: Dimensions, Laws, or Categories? Journal of Personality and Social Psychology, 67, 575-584. https://doi.org/10.1037/0022-3514.67.4.575

Haugaard, M. (2010). Power: A "Family Resemblance" Concept. European Journal of Cultural Studies, 13, 1-20. https://doi.org/10.1177/1367549410377152

Laursen, B., \& Hartup, W. W. (2002). The Origins of Reciprocity and Social Exchange in Friendships. New Directions for Child and Adolescent Development, 95, 27-40. https://doi.org/10.1002/cd.35

Macmurray, J. (1999). Persons in Relation. New York, NY: Humanity Books.

Marx, K. (1859). A Contribution to the Critique of Political Economy. Translated by S. W. Ryazanskaya, London: Lawrence \& Wishart.

McGilchrist, I. (2010). The Master and His Emissary: The Divided Brain and the Making of the Western World. New Haven, CT: Yale University Press.

Plutchik, R. [1962] (1991). The Emotions: Facts, Theories, and a New Model. Lanham, MD: University Press of America.

Rokeach, M. (1960). The Open and Closed Mind: Investigations into the Nature of Belief Systems and Personality Systems. New York, NY: Basic Books.

Rukavishnikov, V. (1992). Public Opinion Structure and Environmental Concerns in Modern Russia. In The "Current Developments in Environmental Sociology" Symposium, Woodshoth (pp. 17-21).

Ryan, R. M., \& Deci, E. L. (2000). The Darker and Brighter Sides of Human Existence: Basic Psychological Needs as a Unifying Concept. Psychological Inquiry, 11, 319-338. https://doi.org/10.1207/S15327965PLI1104_03

Simão, C., \& Seibt, B. (2014). Gratitude Depends on the Relational Model of Communal Sharing. PLoS ONE, 9, e86158. https://doi.org/10.1371/journal.pone.0086158

Stone, W. F. (1986). Personality and Ideology: Empirical Support for Tomkins Polarity 
Theory. Political Psychology, 7, 689-708. https://doi.org/10.2307/3791209

TenHouten, W. D. (2007). A General Theory of Emotions and Social Life. New York, NY: Routledge.

TenHouten, W. D. (2013). Emotion and Reason: Mind, Brain, and the Social Domains of Work and Love. New York, NY: Routledge.

TenHouten, W. D. (2017a) Alienation and Affect. New York, NY: Routledge.

TenHouten, W. D. (2017b). From Primary Emotions to the Spectrum of Affect: An Evolutionary Neurosociology of the Emotions. In A. Ibáñez, L. Sedeño, \& A. M. Garcia (Eds.), Neuroscienceand Social Science: The Missing Link (pp. 141-167). New York, NY: Springer.

TenHouten, W. D. (2017c). Social Dominance Hierarchy and the Pride-Shame System. Journal of Political Power, 10, 94-114. https://doi.org/10.1080/2158379X.2017.1285154

Tomkins, S. S. (1957). Left and Right: A Basic Dimension of Ideology and Personality. In R. W. White (Ed.), The Study of Lives (pp. 388-411). Cambridge, MA: Harvard University Press.

Tönnies, F. (1887). Community and Society (Gemeinschaft und Gesellschaft). New York, NY: Harper \& Row.

van Meter, K. M. (2001). The Structure of Public Opinion Concerning Social Conflicts as a Fractal Structure for Society. International Journal of Computing Anticipatory Systems, 9, 143-158.

van Meter, K. M. (2004). How People See Society: The Network Structure of Public Opinion Concerning Social Conflicts. Connections, 26, 71-89.

van Meter, K. M. (2014). Structures of Human Societies. Sociology Mind, 4, 36-44. https://doi.org/10.4236/sm.2014.41005

Weber, M. (1921). Economy and Society: An Outline of Interpretive Sociology (2 Vols.). Berkeley, CA: University of California Press.

Weinberg, I. (2000). The Prisoners of Despair: Right Hemisphere Deficiency and Suicide. Neuroscience \& Biobehavioral Reviews, 24, 605-626.

https://doi.org/10.1016/S0149-7634(00)00026-9 